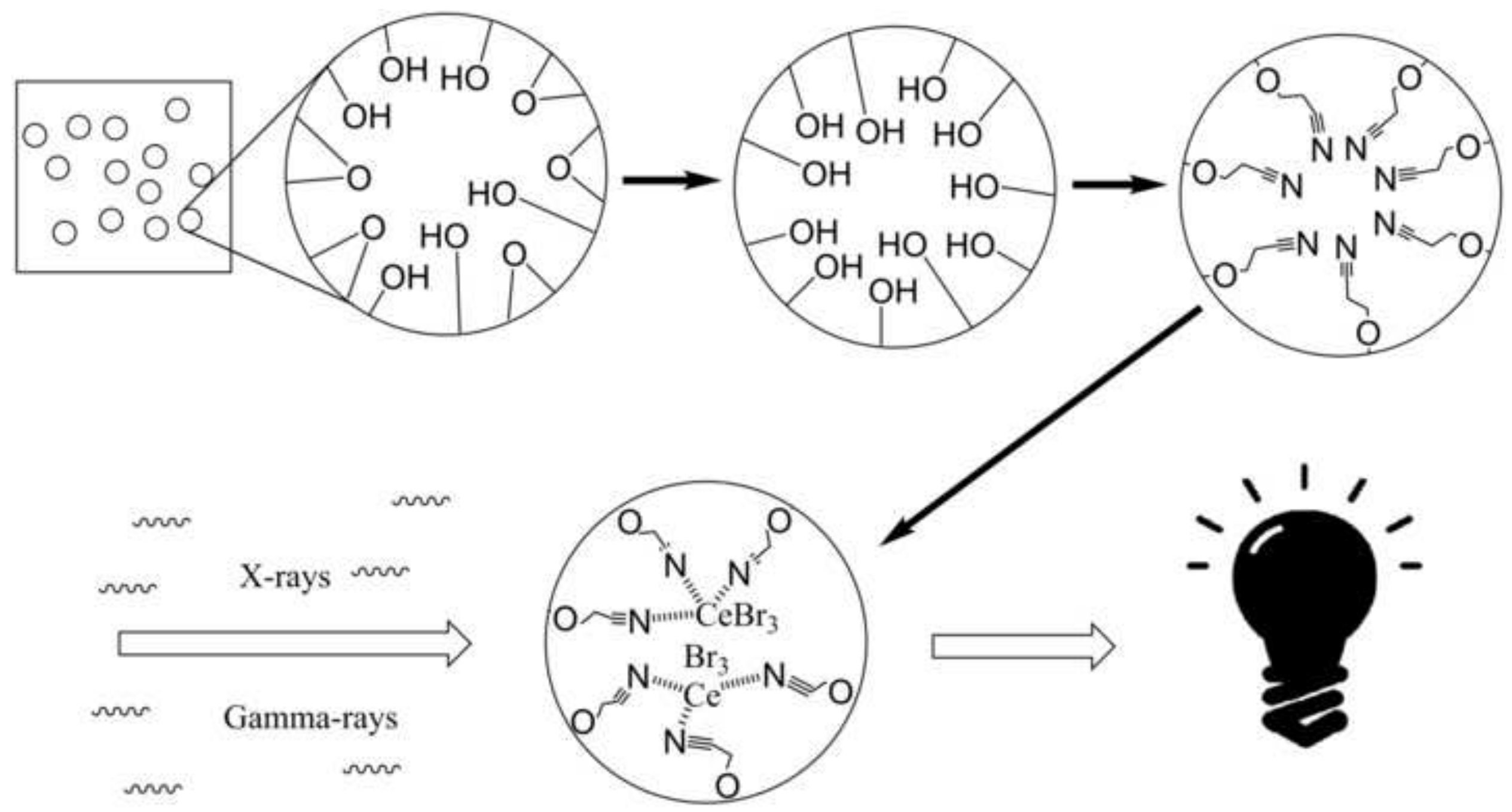

SCINTILLATION! 


\title{
Sequestration of Cerium(III) Bromide into
}

\section{Mesoporous Silica}

\author{
William L. Boncher*, Terri C. Lin ${ }^{I}$, Nikolaus L.Cordes, and Markus P. Hehlen \\ Los Alamos National Laboratory, Materials Science and Technology: Polymers and Coatings \\ Mailstop E549, PO Box 1663, Los Alamos, NM 87545 \\ *Corresponding author. boncher@lanl.gov +1 505-500-7999 \\ ${ }^{1}$ Present Address: Department of Chemistry and Biochemistry, University of California at Los \\ Angeles, CA 90095
}

\begin{abstract}
A variety of surface-functionalized mesoporous silica monoliths were reacted with a cerium(III) bromide molecular complex and a successful scaffold for luminescent materials is demonstrated. The variation in structure of the materials is shown through infrared spectroscopy, and the luminescent properties illustrate the varying coordination environment of cerium(III) bromide.
\end{abstract}

Keywords: cerium bromide, scintillator, mesoporous silica

\section{Introduction}

Single crystals of doped metal halides are widely used as scintillators for gamma-ray detection. They can provide high photon yield and good energy resolution at room temperature. The most 
common material is $\mathrm{NaI}: \mathrm{Tl}[1]$. Recently, lanthanide halide compounds including $\mathrm{LaBr}_{3}: \mathrm{Ce}^{3+}[2]$ and $\mathrm{SrI}_{2}: \mathrm{Eu}^{2+}[3]$ have been developed. They offer the highest photon yields $(65 \mathrm{k}$ photons/MeV for $\mathrm{LaBr}_{3}: \mathrm{Ce}[4]$, and $120 \mathrm{k}$ photons/MeV for $\mathrm{SrI}_{2}: \mathrm{Eu}[5]$, versus $45 \mathrm{k}$ photons/MeV for $\left.\mathrm{NaI}: \mathrm{Tl}[6]\right)$ and energy resolution among room-temperature scintillators, and they enable radiation sensors that can not only detect but also identify the type of source material. However, the growth of large high quality single crystals of these hygroscopic materials is difficult and expensive, and a cheaper alternative method would be very beneficial. Inexpensive organic scintillators such as plastics[7] or liquids[8] have significantly lower photon yields and are not useful for gamma-ray spectroscopy. For this reason, lanthanide halide scintillators that can be fabricated by an inexpensive and scalable method are highly desired.

Nanocomposite scintillators in which nano-sized scintillator particles are contained within an optically transparent matrix, have been the focus of numerous studies[9-11]. The primary challenges in developing a nanocomposite scintillator are 1) achieving a high loading level of the scintillator in order to obtain a gamma attenuation that is competitive with traditional singlecrystal scintillators, and 2) maintaining a high degree of optical transparency (low absorption and low scattering) in order to efficiently transport the scintillation light to the photodetector. The second challenge is particularly difficult due to the high refractive index of the inorganic crystals $(n>2[12-14])$ compared with that of most potential matrices. Silica has an index around 1.5[15], and the resulting large refractive index mismatch between the scintillator and the matrix in a nanocomposite will result in opacity and thus reduced light transport efficiency primarily due to Rayleigh scattering[16]. Particle size has a large impact on scattering[17]; as the scintillator particle size decreases to $\sim 3 \%$ of the wavelength, scattering should become negligible even in the presence of some refractive index mismatch[18]. 
Mesoporous (defined as having pores between 2-50 $\mathrm{nm}[19]$ ) silica was first developed in the 1990s[20] by Mobil, and has been widely used in a variety of applications, such as gas[21] and water[22] treatment, catalysis[23], and even biological applications such as drug delivery[24] and imaging. Mesoporous silica can be modified with a range of functionalities[25], and it has been made with widely tunable properties such as surface area and pore size. Mesoporous silica is an attractive matrix for nanocomposite scintillators because surface functionality can be introduced to bind the scintillator inside the pores. When mesoporous silica is filled with scintillator, the pore size defines the scintillator particle size, which can be sufficiently small to result in potentially low optical scattering.

Our group has recently developed coordination polymers of cerium(III) bromide with ligands coordinated through a nitrile group[26]. Functionalizing the pore surfaces within the mesoporous silica with a nitrile group should allow cerium bromide to bind to the matrix by forming nitrile coordination compounds at the pore surface. Furthermore, we expect that introducing organic ligands will reduce the net refractive index of the composite material (since organic materials typically have low refractive indices) and therefore decrease scattering. The inclusion of organic material however will decrease the possible loading level of cerium(III) bromide and potentially reduce the possible light output. The goal of the present study is to chemically modify mesoporous silica with cerium(III) bromide to introduce scintillation functionality, and assess the luminescent properties.

\section{Experimental}

2.1 Preparation of mesoporous silica (1): Based on a procedure by Zhao[27], $0.8 \mathrm{~g}$ Pluronic F127 was dissolved in $1.0 \mathrm{~g}$ tetraethylorthosilicate, $8 \mathrm{~mL}$ ethanol, and $1 \mathrm{~mL} 0.2 \mathrm{M} \mathrm{HCl}$. The 
solution was stirred at room temperature and pressure for 2 hours and became transparent. The solution was heated at $0.1{ }^{\circ} \mathrm{C} / \mathrm{min}$ to $70{ }^{\circ} \mathrm{C}$, held for 36 hours, and cooled at $0.1{ }^{\circ} \mathrm{C} / \mathrm{min}$ to room temperature. The resulting monolithic gel was then calcined in air to remove the Pluronic by heating it at $1{ }^{\circ} \mathrm{C} / \mathrm{min}$ to $550{ }^{\circ} \mathrm{C}$, holding for 6 hours under ambient pressure, and cooling to room temperature.

2.2 Preparation of hydrolyzed mesoporous silica (2): The mesoporous silica monolith (1) was soaked in a $10 \mathrm{~mL}$ piranha solution $\left(70 \% \mathrm{H}_{2} \mathrm{SO}_{4}, 30 \% \mathrm{H}_{2} \mathrm{O}_{2}\right)$ overnight and then rinsed with water.

\subsection{Preparation of nitrile-functionalized mesoporous silica (3): $1 \mathrm{~mL}$ 3-}

cyanopropyltriethoxysilane was added to $10 \mathrm{~mL}$ toluene. The hydrolyzed mesoporous silica monolith (2) was put in solution and was refluxed for 3 hours (without stirring, to avoid damaging the monolith). The monolith was then rinsed 3 times with toluene, followed by 3 washes with tetrahydrofuran.

2.4 Preparation of $\mathrm{CeBr}_{3} \cdot$ THF solution (4): Based on a method we have previously reported[28], in an argon glovebox, $0.1 \mathrm{~g} \mathrm{CeBr}_{3}$ was heated in $10 \mathrm{~mL}$ tetrahydrofuran at $50{ }^{\circ} \mathrm{C}$ while stirring overnight, then filtered through a coarse glass frit.

2.5 Preparation of mesoporous silica sequestered $\mathrm{CeBr}_{3}(5)$ : In an argon glovebox, a mesoporous silica monolith (1) was heated overnight in $\mathrm{CeBr}_{3} \cdot \mathrm{THF}$ solution (4) at $50{ }^{\circ} \mathrm{C}$, then dried on a watchglass. The monolith remained intact, and the same in appearance to the starting material. 
2.6 Preparation of hydrolyzed mesoporous silica sequestered $\mathrm{CeBr}_{3}(6)$ : In an argon glovebox, a hydrolyzed mesoporous silica monolith (2) was heated overnight in $\mathrm{CeBr}_{3} \cdot \mathrm{THF}$ solution (4) at $50{ }^{\circ} \mathrm{C}$, then dried on a watchglass. The monolith remained intact, and the same in appearance to the starting material.

2.7 Preparation of nitrile-functionalized mesoporous silica sequestered $\mathrm{CeBr}_{3}(7)$ : In an argon glovebox, a nitrile-functionalized mesoporous silica monolith (3) was heated overnight in $\mathrm{CeBr}_{3} \cdot \mathrm{THF}$ solution $(4)$ at $50{ }^{\circ} \mathrm{C}$, then dried on a watchglass. The monolith remained intact, and the same in appearance to the starting material.

2.8 Characterization: Fourier Transform Infrared (FT-IR) spectra of the samples in KBr pellets were measured using a Bruker Vertex $80 \mathrm{~V}$ FT-IR spectrometer at $4 \mathrm{~cm}^{-1}$ energy resolution. Photoluminescence spectra were measured using a Photon Technologies International Timemaster photoluminescence spectrophotometer. Nitrogen physisorption was performed using a Quantachrome Autosorb-1 instrument at $-196{ }^{\circ} \mathrm{C}$. Samples were outgassed under vacuum at $523.15 \mathrm{~K}$ for at least 48 hours prior to analysis. Specific surface areas were calculated according to Brunauer-Emmett-Teller (BET) theory by taking a minimum of five data points of the adsorption branch within the range of 0.05 to 0.30 relative pressures $\left(\mathrm{P} / \mathrm{P}_{0}\right)$. Pore size distributions were calculated according to Barrett-Joyner Halenda (BJH) theory using the desorption branch, as well as the equilibrium model for a cylindrical pore shape in silica, based off of non-local density functional theory (NLDFT) calculations. Radioluminescence (RL) spectroscopy was performed using a homemade apparatus featuring a charge-coupled device (CCD) (Jobin-Yvon Spectrum One 3000) coupled to a Jobin-Yvon Triax 180 monochromator as the detection system. Sample excitation was obtained by X-ray irradiation through a Be window, using a Phillips $2274 \mathrm{X}$-ray tube with a tungsten target operated at $50 \mathrm{kV}$ and producing an 
effective X-ray energy of $\sim 25 \mathrm{keV}$ with a dose rate of $1.75 \mathrm{~Gy} / \mathrm{s}$ for 30 seconds. The data was corrected for the spectral response of the detection system.

\section{Results and Discussion}

3.1 BET. A typical nitrogen physisorption isotherm of a mesoporous silica monolith is shown in Figure 1. The isotherm exhibits a fast rate of adsorption in the relative pressure $\left(\mathrm{P} / \mathrm{P}_{0}\right)$ range of 0.05-0.60 before reaching a steady-state of adsorption in the $\mathrm{P} / \mathrm{P}_{0}$ range of $\sim 0.6-0.95$. At $\mathrm{P} / \mathrm{P}_{0}>0.95$, an uptake is observed that indicates macroporosity. BET specific surface area (SSA) derived from the nitrogen physisorption isotherm (Figure 1) is presented in Table 1. The pore size distribution presented in Figure 1, derived from the isotherm, exhibits a narrow monomodal peak at $3.43 \mathrm{~nm}$, which can be attributed to surfactant templating. The pore size distribution as determined from NLDFT shows a larger pore size, with a peak at $4.74 \mathrm{~nm}$ is in close enough agreement with the $\mathrm{BJH}$ data, given that $\mathrm{BJH}$ can underestimate pore size by $20-30 \%$ (28\% here) for pores smaller than $10 \mathrm{~nm}$.[29,30] Given the derived total pore volume and assuming a silica density of $2.65 \mathrm{~g} \mathrm{~cm}^{-3}[31]$, the pore volume fraction is calculated to $54.9 \pm 0.1 \%$. Due to the chemical instability of surface-modified mesoporous silicas at the high outgassing temperature $\left(250^{\circ} \mathrm{C}\right)$, nitrogen physisorption isotherms acquired after silica modification were unsuitable for analysis. Outgassing at lower temperatures for longer times was also ineffective at degassing without decomposition of the material.

Table 1. Specific surface area and pore diameter of mesoporous silica monolith (1) derived from nitrogen physisorption data. ${ }^{a}$

\begin{tabular}{ccccc}
\hline & $\begin{array}{c}\text { BET Specific } \\
\text { Surface Area } \\
\left(\mathrm{m}^{2} \mathrm{~g}^{-1}\right)\end{array}$ & $\begin{array}{c}\text { BJH Pore } \\
\text { Diameter }(\mathrm{nm})\end{array}$ & $\begin{array}{c}\text { NLDFT Pore } \\
\text { Diameter }(\mathrm{nm})\end{array}$ & $\begin{array}{c}\text { Total Pore Volume } \\
\left(\mathrm{cm}^{3} \mathrm{~g}^{-1}\right)^{b}\end{array}$ \\
\hline $\mathbf{1}$ & $478 \pm 18$ & $3.43 \pm 0.01$ & $4.74 \pm 0.30$ & $4.60 \times 10^{-1} \pm 2.0 \times 10^{-3}$ \\
\hline
\end{tabular}


${ }^{a}$ Values are the average measurements of 3 samples. Uncertainty is the standard deviation of the average.

${ }^{b}$ Total pore volume for pores smaller than 3.2 micrometers at $\mathrm{P} / \mathrm{P}_{0}=0.9994$

Figure 1. Left: Typical nitrogen physisorption isotherm of a mesoporous silica monolith. Right: Typical pore size distribution (derived from the isotherm) of a mesoporous silica monolith, exhibiting a narrow monomodal peak at $3.43 \mathrm{~nm}$, via BJH desorption, and $4.74 \mathrm{~nm}$ by NLDFT calculations.

3.2 Infrared Spectroscopy. A monolith of mesoporous silica was made and the pores were functionalized with a nitrile group in an attempt to facilitate the sequestration of cerium bromide. IR spectroscopy was used to follow the various synthesis steps, as shown in Figure 2. First, the silica monolith (1) was hydrolyzed in piranha solution to increase the number of reactive $\mathrm{Si}-\mathrm{OH}$ bonds on the pore surfaces. This can be seen by the increased intensity of the mode at $1630 \mathrm{~cm}^{-1}$ due to the $\mathrm{O}-\mathrm{H}$ bending vibration. This hydrolyzed piece (2) was reacted with a siloxane compound containing a nitrile functional group (3-cyanopropyltriethoxysilane). The inset shows the associated nitrile stretching mode at $2260 \mathrm{~cm}^{-1}$ as well as the methylene stretching mode (from the added siloxane ligand) at $2920 \mathrm{~cm}^{-1}$ now present in $\mathbf{3}$, indicating that the surface had been nitrile functionalized.

Figure 2. IR spectra of mesoporous silica (1), hydrolyzed mesoporous silica (2), nitrile-functionalized mesoporous silica (3), and $\mathrm{CeBr}_{3}$ sequestered in nitrile-functionalized mesoporous silica. The inset shows the detail of the spectral region featuring the methylene and nitrile groups from the 3cyanopropyltriethoxysilane addition. 
Unexpectedly, once the nitrile-functionalized sample (3) was reacted with the $\mathrm{CeBr}_{3} \cdot \mathrm{THF}$ solution, the sample (7) no longer showed the same methylene or nitrile stretching modes as seen in 3 . This indicates that the nitrile functionality was displaced rather than employed by the $\mathrm{CeBr}_{3}$ to form a surface coordination complex. However, cerium is clearly present in $\mathbf{7}$ as evidenced by the luminescence properties (see below). Based on this, we adjusted our approach to also attempt sequestering $\mathrm{CeBr}_{3}$.THF in the silica monolith (1) and the hydrolyzed monolith $(\mathbf{2}-)_{-}$, as the nitrile group does not seem to be playing the role we expected.

3.3 Luminescence. Photoluminescence measurements are shown in Figure 3, which show the photoluminescence excitation (PLE) and photoluminescence (PL) spectra of the three infused samples (5-7), as well as the spectra of neat $\mathrm{CeBr}_{3}$ and the $\mathrm{CeBr}_{3}$. THF complex used in the syntheses (4). These materials are also radioluminescent, as expected, since $\mathrm{CeBr}_{3}$ is a known scintillator; the radioluminescence spectra are shown in the in Figure 4.

Figure 3. Photoluminescence excitation (PLE) and emission (PL) spectra of the $\mathrm{CeBr}_{3}$ infused samples (57), as well as neat $\mathrm{CeBr}_{3}$ and the $\mathrm{CeBr}_{3} \cdot \mathrm{THF}$ complex (4).

Figure 4. Radioluminescence (RL) spectra of the $\mathrm{CeBr}_{3}$ infused samples (5-7), as well as neat $\mathrm{CeBr}_{3}$ and the $\mathrm{CeBr}_{3} \cdot \mathrm{THF}$ complex (4).

The $\mathrm{Ce}^{3+}$ luminescence is a sensitive probe of coordination environment, so the differences in structure of the infused materials can be observed in the PLE/PL spectra. The shift in the PLE/PL 
between $\mathbf{6}$ and $\mathbf{7}$ is subtle $(\sim 5 \mathrm{~nm})$, suggesting similar coordination, whereas 5 is more similar to neat $\mathrm{CeBr}_{3}$ than 6 or 7 .

The RL spectra in Figure 4 correlate with the PL spectra, demonstrating the response of these materials to high-energy radiation, just as cerium(III) bromide does.

Optically, the final monoliths containing cerium(III) bromide remained opaque. This is most likely due to the pores being incompletely filled with $\mathrm{CeBr}_{3}$, leaving residual gas-filled voids that represent a large refractive index mismatch and thus cause excessive optical scattering. This is problematic for applications that require large-volume scintillators. In an opaque scintillator the net light output will be significantly lower since only photons generated on or near the surface are able to escape the material.

The difference in the PL/PLE and the RL spectra between the various samples demonstrates that the surface of the silica pore has a strong impact on the resulting luminescent properties. It is also noteworthy that all of the infused samples have similar light outputs. Because all of the silica samples we used were able to be infused regardless of their surface functionalization, it is expected that a wide range of fine-tunable luminescent materials could be made, depending on the specific luminescent properties which are needed.

\section{Conclusions}

We have demonstrated that mesoporous silica can be functionalized with a scintillating material. The scintillator largely maintains its scintillation properties, with small variations in the emission wavelength due to the coordination environment at the pore surface. The resulting material is not transparent, likely due to incomplete pore filling, which currently limits its application as a 
replacement for scintillation crystals. Efforts are underway to increase the transparency of the material by completely filling the pores with $\mathrm{CeBr}_{3}$. Regardless of this outcome, the methodology developed here can be applied to produce finely tunable luminescent nanocomposite materials in a monolithic scaffold.

\section{Acknowledgements}

This work is supported by the Department of Energy (DOE) Los Alamos National Laboratory (LANL) Laboratory Directed Research and Development (LDRD) program, 20120246ER.

\section{References}

[1] M. Gascón, S. Lam, S. Wang, S. Curtarolo, R.S. Feigelson, Radiat. Meas. 56 (2013) 70.

[2] E.V.D. van Loef, P. Dorenbos, C.W.E. van Eijk, K. Kra $\square$ mer, H.U. Gu $\square$ del, Appl. Phys. Lett. 77 (2000) 1467.

[3] K. Yang, M. Zhuravleva, C.L. Melcher, J. Lumin. 132 (2012) 1824.

[4] P.R. Menge, G. Gautier, A. Iltis, C. Rozsa, V. Solovyev, Nucl. Instrum. Methods Phys. Res., Sect. A 579 (2007) 6.

[5] E. V. van Loef, C.M. Wilson, N.J. Cherepy, G. Hull, S.A. Payne, W. Choong, W.W. Moses, K.S. Shah, IEEE Trans. Nucl. Sci. 56 (2009) 869.

[6] E. Sakai, IEEE Trans. Nucl. Sci. NS-34 (1987) 418.

[7] N. Zaitseva, B.L. Rupert, I. PaweŁczak, A. Glenn, H.P. Martinez, L. Carman, M. Faust, N. Cherepy, S. Payne, Nucl. Instrum. Methods Phys. Res., Sect. A 668 (2012) 88.

[8] A.A. Naqvi, A. Aksoy, F.Z. Khiari, A. Coban, M.M. Nagadi, M.A. Al-Ohali, M.A. AlJalal, Nucl. Instrum. Methods Phys. Res., Sect. A 345 (1994) 514.

[9] N.Z. Galunov, B. V Grinyov, N.L. Karavaeva, J.K. Kim, Y.K. Kim, O.A. Tarasenko, E. V Martynenko, IEEE Trans. Nucl. Sci. 56 (2009) 904.

[10] M. V. Astakhov, G.S. Belanov, V.G. Vasil'chenko, a. O. Rodin, V.D. Samoilenko, a. S. Solov'ev, Inst. Exper. Tech. 49 (2006) 645. 
[11] M. Nikl, N. Solovieva, K. Apperson, D.J.S. Birch, A. Voloshinovskii, Appl. Phys. Lett. 86 (2005) 101914.

[12] D.J. Singh, Appl. Phys. Lett. 92 (2008) 201908.

[13] A. Iltis, M.R. Mayhugh, P. Menge, C.M. Rozsa, O. Selles, V. Solovyev, Nucl. Instrum. Methods Phys. Res., Sect. A 563 (2006) 359.

[14] H.T.. van Dam, S. Seifert, W. Drozdowski, P. Dorenbos, D.R. Schaart, IEEE Trans. Nucl. Sci. 59 (2012) 656.

[15] I.H. Malitson, J. Opt. Soc. Amer. 55 (1965) 1205.

[16] H. Althues, J. Henle, S. Kaskel, Chem. Soc. Rev. 36 (2007) 1454.

[17] E.A. McKigney, R.E. Del Sesto, L.G. Jacobsohn, P.A. Santi, R.E. Muenchausen, K.C. Ott, T. Mark McCleskey, B.L. Bennett, J.F. Smith, D.W. Cooke, Nucl. Instr. Meth. Phys. Res. A 579 (2007) 15.

[18] R. Fedyk, D. Hreniak, W. Łojkowski, W. Stręk, H. Matysiak, E. Grzanka, S. Gierlotka, P. Mazur, Opt. Mater. 29 (2007) 1252.

[19] K.S.W. Sing, D.H. Everett, R.A.W. Haul, L. Moscou, R.A. Pierotti, J. Rouquerol, T. Siemieniewska, REPORTING PHYSISORPTION DATA FOR GAS / SOLID SYSTEMS with Special Reference to the Determination of Surface Area and Porosity, 1985.

[20] J.S. Beck, K.D. Schmitt, J.B. Higgins, J.L. Schlenkert, J. Am. Chem. Soc. 114 (1992) 10834.

[21] A. Fukuoka, J. Kimura, T. Oshio, Y. Sakamoto, M. Ichikawa, J. Am. Chem. Soc. 129 (2007) 10120.

[22] X. Feng, G.E. Fryxell, L.-Q. Wang, A.Y. Kim, J. Liu, K.M. Kemner, Science 276 (1997) 923.

[23] A. Corma, Chem. Rev. 97 (1997) 2373.

[24] M. Vallet-Regi, A. Rámila, R.P. del Real, J. Pérez-Pariente, Chem. Mater. 13 (2001) 308.

[25] M. Park, S. Komarneni, Microporous Mesoporous Mater. 25 (1998) 75.

[26] K. V. Vasudevan, W.L. Boncher, N. a. Smith, M.W. Blair, B.L. Scott, B.L. Bennett, M.P. Hehlen, R.E. Muenchausen, J.C. Gordon, Eur. J. Inorg. Chem. (2014) 2213.

[27] H. Wei, Y. Lv, L. Han, B. Tu, D. Zhao, Chem. Mater. 23 (2011) 2353. 
[28] K. V. Vasudevan, N.A. Smith, B.L. Scott, E.A. McKigney, M.W. Blair, J.C. Gordon, R.E. Muenchausen, Inorg. Chem. 50 (2011) 4627.

[29] M. Thommes, Chem. Ing. Tech. 82 (2010) 1059.

[30] M. Thommes, in:, Nanoporous Mater. Sci. Eng., 2004, p. 317.

[31] W.M. Hayes, CRC Handbook of Chemistry and Physics, 2012. 


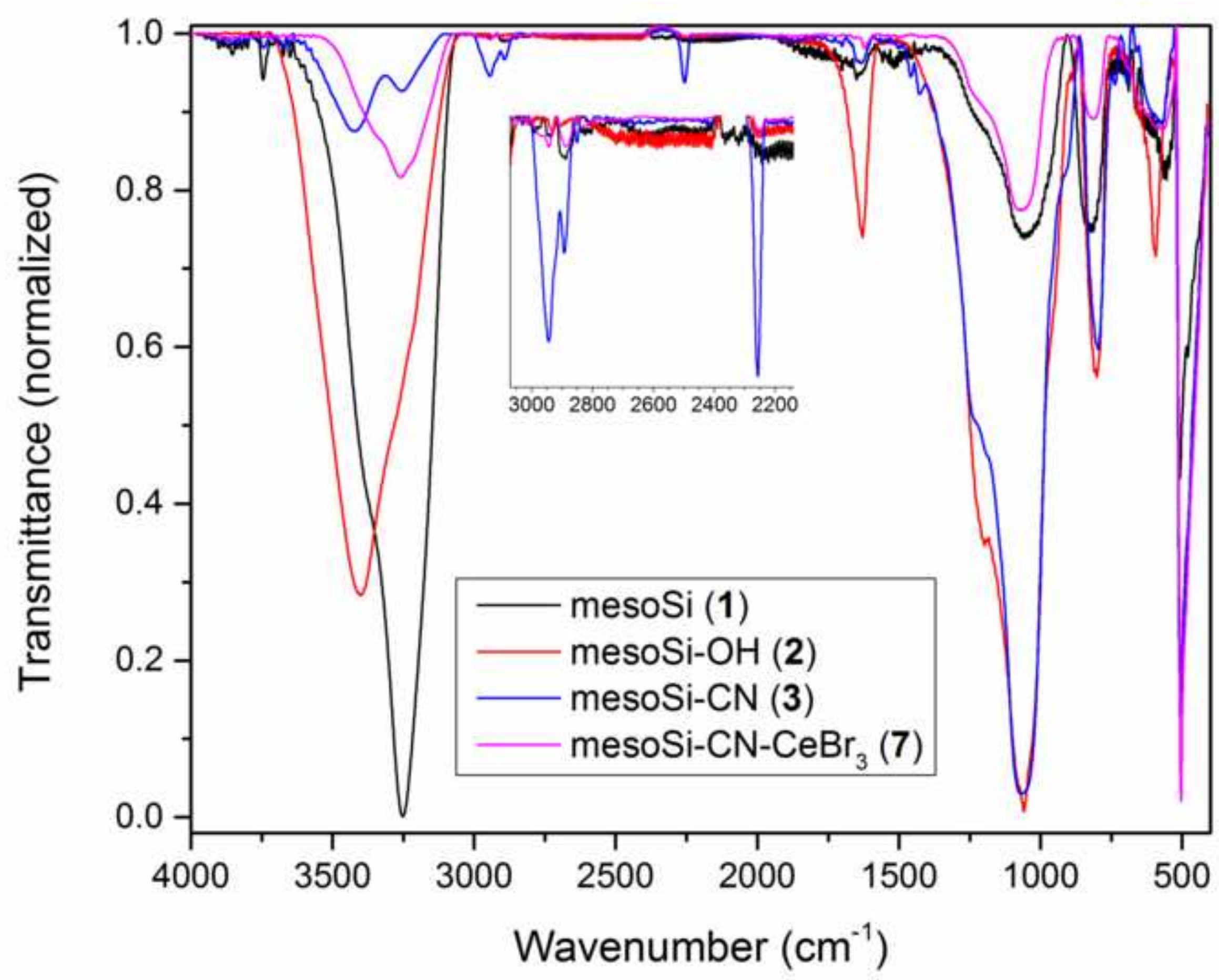



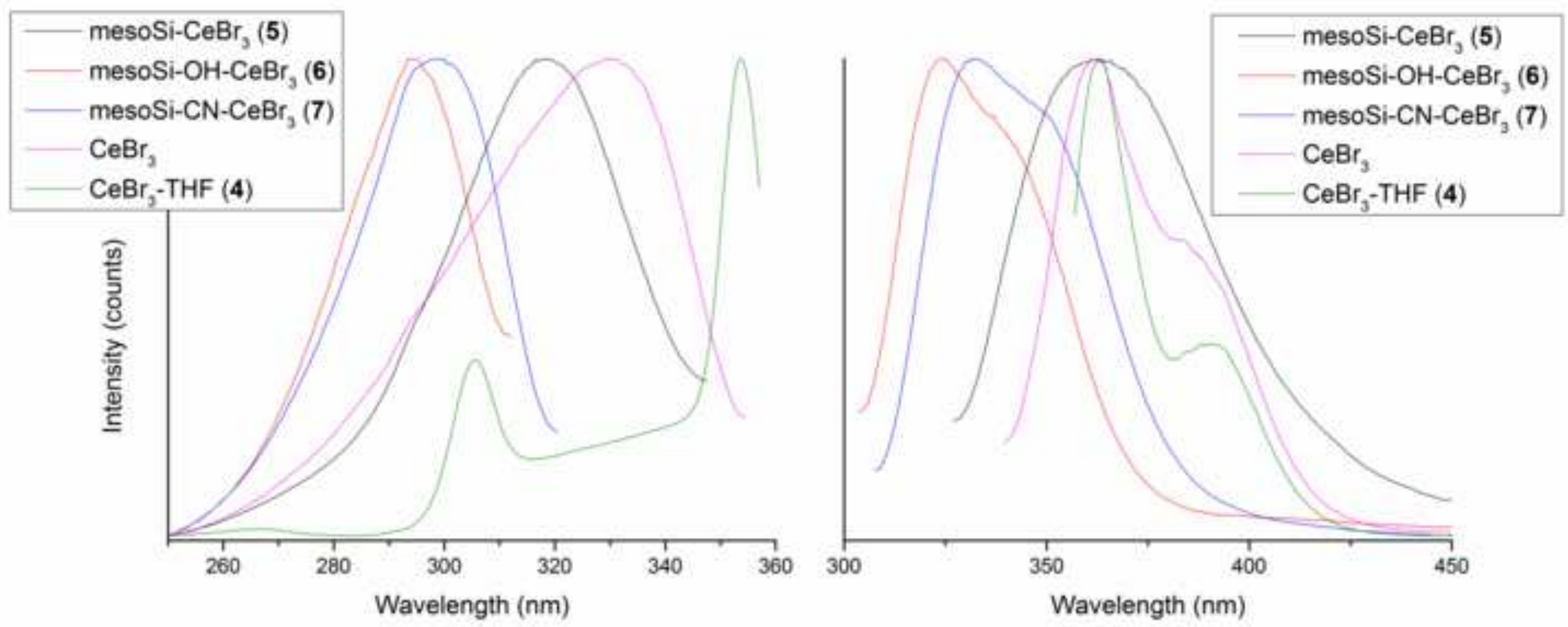


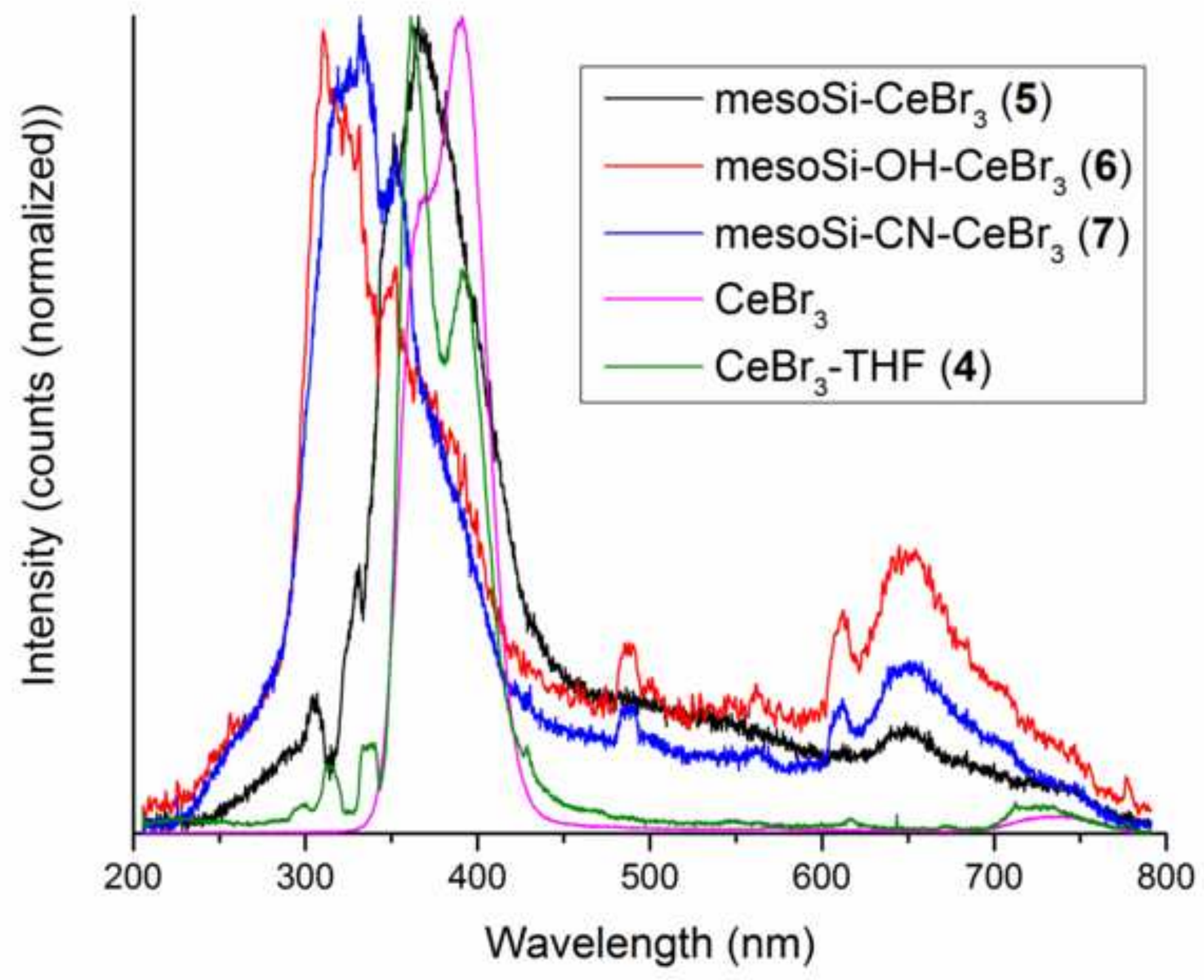



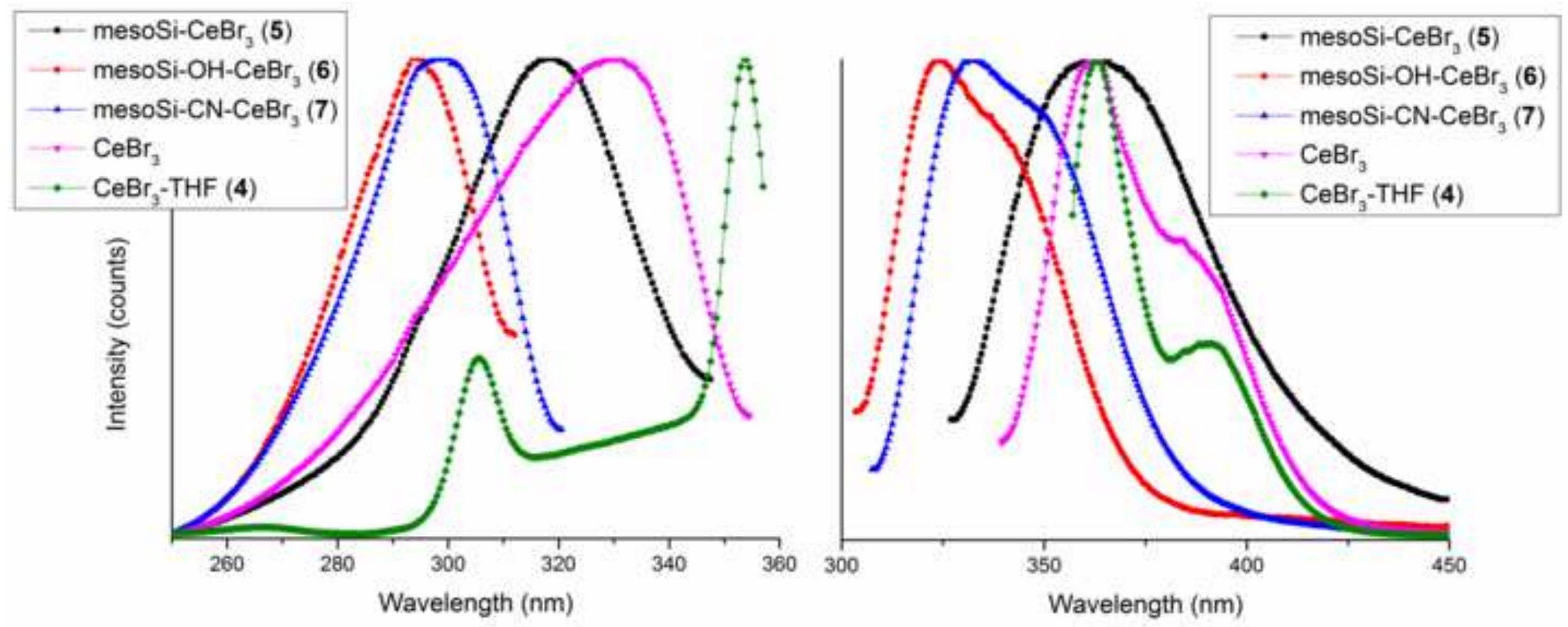


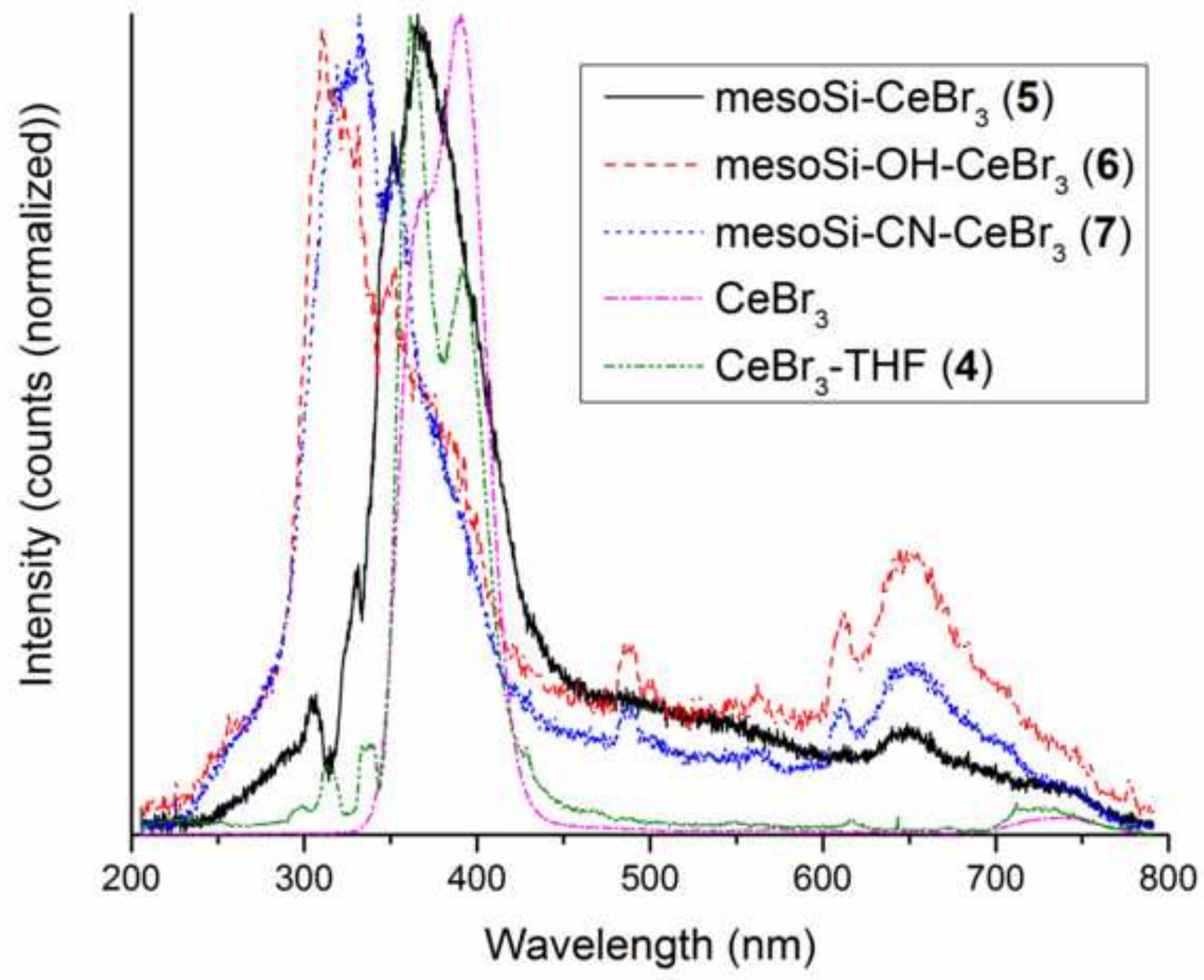




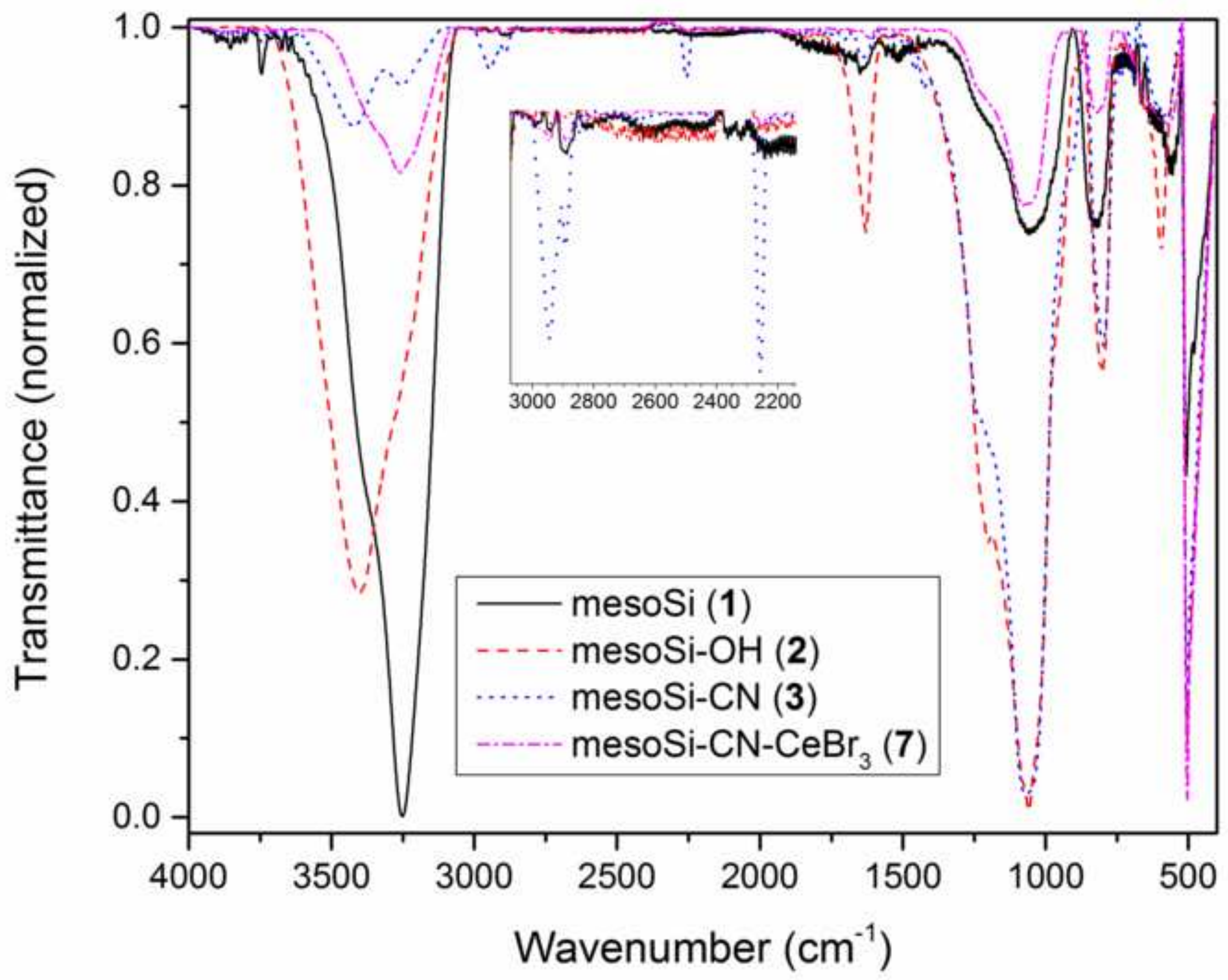



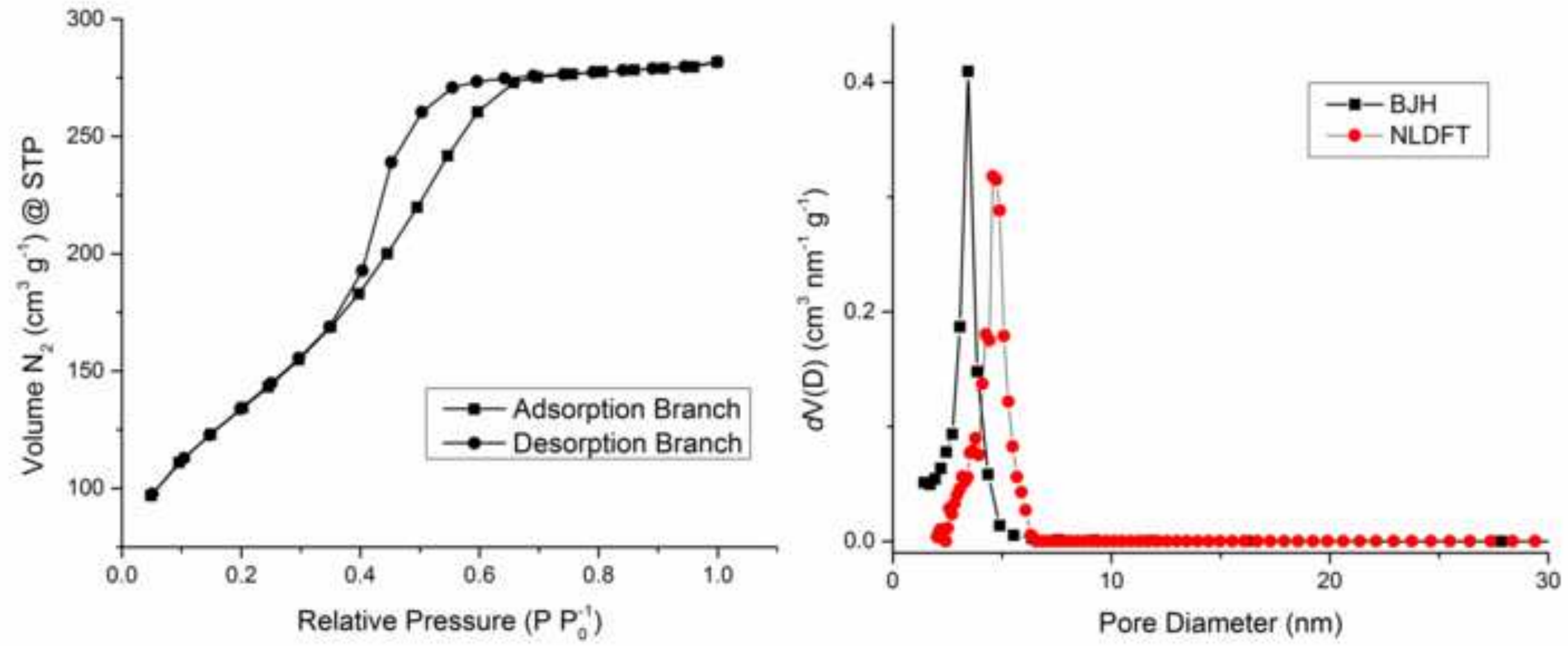